\title{
PercepFiguras: programa computarizado de estimulación visual para evaluar conductual y electrofisiológicamente la percepción multiestable
}

\section{PercepFiguras: a computerized visual stimulation program to assess, behaviorally and electrophysiologically, the multistable perception}

\author{
E. E. Ortiz-Cruz', H. Rodríguez-Camacho', M. A. Rodríguez-Camacho', J. Bernal-Hernández² \\ 'Facultad de Estudios Superiores de Iztacala, UNAM, México \\ ${ }^{2}$ Facultad de Estudios Superiores de Iztacala, UNAM, México; Instituto de Neuropsicología y Neurociencias Cognitivas
}

\section{RESUMEN}

En la investigación de la percepción visual comúnmente se usan programas computarizados comerciales para la presentación de los estímulos, configurados por lo general para admitir una respuesta por cada estímulo presentado. Sin embargo, el estudio de la percepción multiestable, requiere la presentación de un estímulo que permanece invariable aunque la percepción de éste puede variar en dos o más configuraciones, lo que demanda que el programa admita respuestas para cada cambio en la percepción de un mismo estímulo. Se presenta PercepFiguras desarrollado con lenguaje de programación C++ que es un sistema de estimulación visual que permite al usuario: a) presentar imágenes y recibir una o varias respuestas a cada una, b) sincronizar las respuestas con un sistema de registro electroencefalográfico mediante el envío de pulsos TTL, c) generar un archivo conductual y d) generar un archivo con el total de respuestas y promedios de las latencias a cada tipo de figura. Se realizó un estudio piloto con una tarea de presentación continua de una figura ambigua alternando con dos variantes de menor ambigüedad, se obtuvo mayor frecuencia de cambios perceptuales y menor estabilidad perceptual para la figura ambigua respecto a las de menor ambigüedad, comprobándose la utilidad del programa.

PALABRAS CLAVE: cubo de Necker, figura ambigua, presentación continua, respuestas variables, sistema computacional 


\section{ABSTRACT}

In the study of visual perception, the use of computerized commercial programs for the presentation of stimuli, usually configured to admit a response for each stimulus presented, is common. However, the study of multistable perception requires the presentation of a stimulus that remains unchanged, although the perception of it can vary in two or more configurations during its observation, which demands that the program admit responses for each change in perception of the same stimulus. PercepFiguras is a system developed with C++ programming language that allows: a) present images and receive multiple responses to the same stimulus, b) synchronize the responses with an electroencephalographic recording system by sending TTL pulses, c) generate a behavioral file, d) generate a file with the total responses and averages of the latencies for each type of figure. In the pilot study with a task of continuous presentation of an ambiguous figure to alternating with two variants of less ambiguity, we observe greater frequency of perceptual and less perceptual stability to the ambiguous figure with respect to the less ambiguous figures, which proves the usefulness of the program.

KEYWORDS: Necker cube, ambiguous figure, continuous presentation, variable responses, computer system

\section{Correspondencia}

DESTINATARIO: Jorge Bernal Hernández

INSTITUCIÓN: Facultad de Estudios Superiores de

Iztacala, UNAM, México

DIRECCIÓN: Av. de los Barrios \#1, Los Reyes Iztacala,

Tlalnepantla, C. P. 54090, Estado de México, México

CORREO ELECTRÓNICO: jbernal@unam.mx

\section{Fecha de recepción:}

15 de enero del 2018

Fecha de aceptación:

12 de abril del 2018 


\section{INTRODUCCIÓN}

Cuando los estímulos presentan características ambiguas, el sistema visual alterna espontáneamente entre dos o más interpretaciones posibles, a pesar de que el estímulo físico siga siendo el mismo; a este fenómeno se le conoce como percepción multiestable. Un ejemplo claro ocurre con las figuras ambiguas, en donde el sujeto puede percibir de dos o más formas diferentes un estímulo que no cambia. Tales percepciones se alternan en el tiempo, dando lugar a cambios perceptuales espontáneos ${ }^{[1]}$.

Una de las figuras ambiguas más utilizada en el estudio de la percepción multiestable es el "cubo de Necker" ${ }^{[2]}$, donde la orientación percibida de la profundidad de la cara principal del cubo tiene dos posibles interpretaciones (orientada a la derecha o a la izquierda) que se alternan en el tiempo. Se ha descrito que alrededor de cada 4 segundos se reporta un cambio perceptual en la orientación del cubo ${ }^{[3]}{ }^{[4]}$. La frecuencia de los cambios perceptuales puede ser influenciada por el modo de presentación de los estímulos. De esta forma, cuando la figura se presenta de manera discontinua, se observa una disminución de los cambios perceptuales conforme más corto es el tiempo interestímulo ${ }^{[5]}$.

El interés principal al estudiar la percepción multiestable es explicar los procesos cognoscitivos responsables de la ocurrencia de los cambios perceptuales espontáneos; en general se habla de una interacción entre las características físicas de los estímulos y los procesos de control voluntario ${ }^{[6]}$, tal como la atención y la memoria. Estas conclusiones se derivan a partir de los resultados de respuestas conductuales (frecuencia de cambios y duración de la percepción). No obstante los resultados conductuales no logran dar una explicación satisfactoria sobre los procesos cognoscitivos responsables del cambio perceptual espontáneo. Por esta razón, se ha incursionado en el estudio de la actividad eléctrica cerebral presente cuando sucede un cambio perceptual espontáneo al observar figuras ambiguas. Esto permitiría describir los procesos jerárquicos que dan lugar a la conciencia perceptual de dichos cambios. Por otra parte, en el área clínica permitiría investigar las alteraciones del funcionamiento cerebral en padecimientos que incluyen alteraciones perceptuales, como la esquizofrenia y los trastornos del espectro autista. Específicamente, en el caso de la esquizofrenia se han observado fallas en el mantenimiento de la coherencia perceptual, los pacientes dependen en mayor medida de la información sensorial, respecto a personas sanas ${ }^{[7]}$. Es posible que esta característica se pueda utilizar como un indicador temprano para detectar esta entidad patológica.

Para el estudio de este fenómeno, es necesario el uso de programas computarizados de estimulación visual que puedan sincronizarse con los registros electrofisiológicos, y brinden precisión en la medición del tiempo de duración de los estímulos, así como en la medición de la latencia y el número de respuestas en la unidad de tiempo. Para lograr el registro sincronizado de la presentación de los estímulos y las respuestas motoras con la actividad cerebral asociada a estos sucesos, es importante que los programas marquen en el registro electrofisiológico el momento de aparición del estímulo y el momento en que los participantes responden, permitiendo así el estudio conductual y electrofisiológico simultáneo de este fenómeno perceptual.

La mayoría de las investigaciones al respecto han utilizado el método de presentación discontinua, donde el estímulo se presenta intermitentemente en periodos cortos de tiempo $(800 \mathrm{~ms})$, con tiempos interestímulo igualmente cortos (de $100 \mathrm{~ms}$ a $500 \mathrm{~ms}$ ). En este tipo de tareas se pide al participante indicar, si cuando se le presenta un estímulo (e.g un cubo de Necker), la orientación en la que lo percibe es igual o diferente al anterior [8] [9]. Sin embargo, el uso de duraciones cortas del estímulo y el aumento del intervalo interestímulo, reduce los cambios espontáneos en la percepción ${ }^{[10]}$. Estas 
últimas características requieren mayor demanda atencional, además de comprometer procesos de memoria, al requerir la comparación de la imagen previa con la actual, para tomar una decisión y dar una respuesta ${ }^{[11]}$.

Estas limitaciones se eliminan utilizando programas de presentación continua de estímulos con figuras que permanecen invariables. En dichos estudios se presenta una imagen ambigua que permanece estable por periodos de tiempo relativamente prolongados (de $1 \mathrm{a}$ 6 minutos), instruyendo al sujeto que indique cada vez que perciba espontáneamente un cambio en la orientación del cubo ${ }^{[12]}{ }^{[13]}$. No obstante, la presentación de estímulos por períodos muy prolongados provoca movimientos oculares y parpadeos, que inducen por sí mismos el cambio perceptual ${ }^{[14]}$, y provocan artefactos eléctricos que dificultan el análisis confiable de la actividad eléctrica cerebral. Por lo que se puede inferir que utilizando períodos de presentación de estímulos, inferiores al intervalo de 1-6 minutos es posible asegurar suficientes épocas de análisis libres de artefactos oculares que distorsionan el registro electrofisiológico.

Otra característica deseable de los paradigmas de percepción multiestable, es que permitan la presentación de figuras control, que induzcan el cambio perceptual (por ejemplo hacia una posición determinada en el cubo de Necker). De esta manera, se podría comparar la conducta y la actividad cerebral asociadas al cambio perceptual espontáneo con las asociadas al cambio inducido. Sin embargo, de los artículos citados en el presente trabajo, sólo se encontraron 5 que usaron este tipo de figuras inductoras. Algunos las presentan alternadas con la figura ambigua, pero con duraciones diferentes [14] [15] [16] 17], mientras que otros lo hacen en bloques de presentación separados ${ }^{[13]}$.

Si bien el diseño de las tareas se puede realizar con programas de acceso libre como Psychtoolbox ${ }^{[18]}$, éste utiliza un conjunto de funciones en Matlab, que requiere del usuario cierto conocimiento en programa- ción. También puede utilizarse el PTT [19], que permite la creación de figuras y brinda precisión en los tiempos de presentación, sin embargo éste no muestra información clara sobre la presentación de los datos de latencia delasrespuestas. Igualmente, el programa "MemPavox" permite la presentación de palabras o de cualquier otro estímulo visual; no obstante, se desarrolló con un objetivo específico para la evaluación de la memoria de trabajo y en la actualidad no se encuentra disponible en la web para valorar sus características ${ }^{[20]}$.

Por otra parte, el uso de sistemas de estimulación (e.g. STIM de la compañía Compumedics) que complementan los programas de registro electrofisiológico como el NeuroSCAN o Curry (de la misma compañía) están diseñados para usarse en el estudio de los correlatos de la actividad eléctrica cerebral asociada a diversos procesos cognoscitivos, en donde no es necesario el registro de varias respuestas a un mismo estímulo, por lo que con estos programas, resulta difícil elaborar una tarea con las características mencionadas anteriormente. Estos programas son herramientas de cuarta generación, que permiten al usuario hacer una presentación de estímulos mediante la elección de instrucciones computacionales preestablecidas que definen la presentación de cada estímulo, al cual le corresponde exclusivamente una respuesta. De acuerdo a nuestra experiencia, este problema podría resolverse en el programa STIM presentando $\mathrm{n}$ veces la misma figura con una duración más corta ( $1 / \mathrm{n}$ de la duración requerida) hasta completar la duración deseada, para poder recibir así una respuesta en cada uno de ellos. Sin embargo, esta solución requiere por parte del investigador, de complicadas manipulaciones adicionales de los archivos de datos conductuales y de registro.

El objetivo del presente trabajo fue desarrollar un programa flexible de estimulación visual para el estudio de la percepción multiestable (llamado PercepFiguras) que pueda ser usado libremente por investigadores en el área de la salud interesados en la percepción multiestable. 


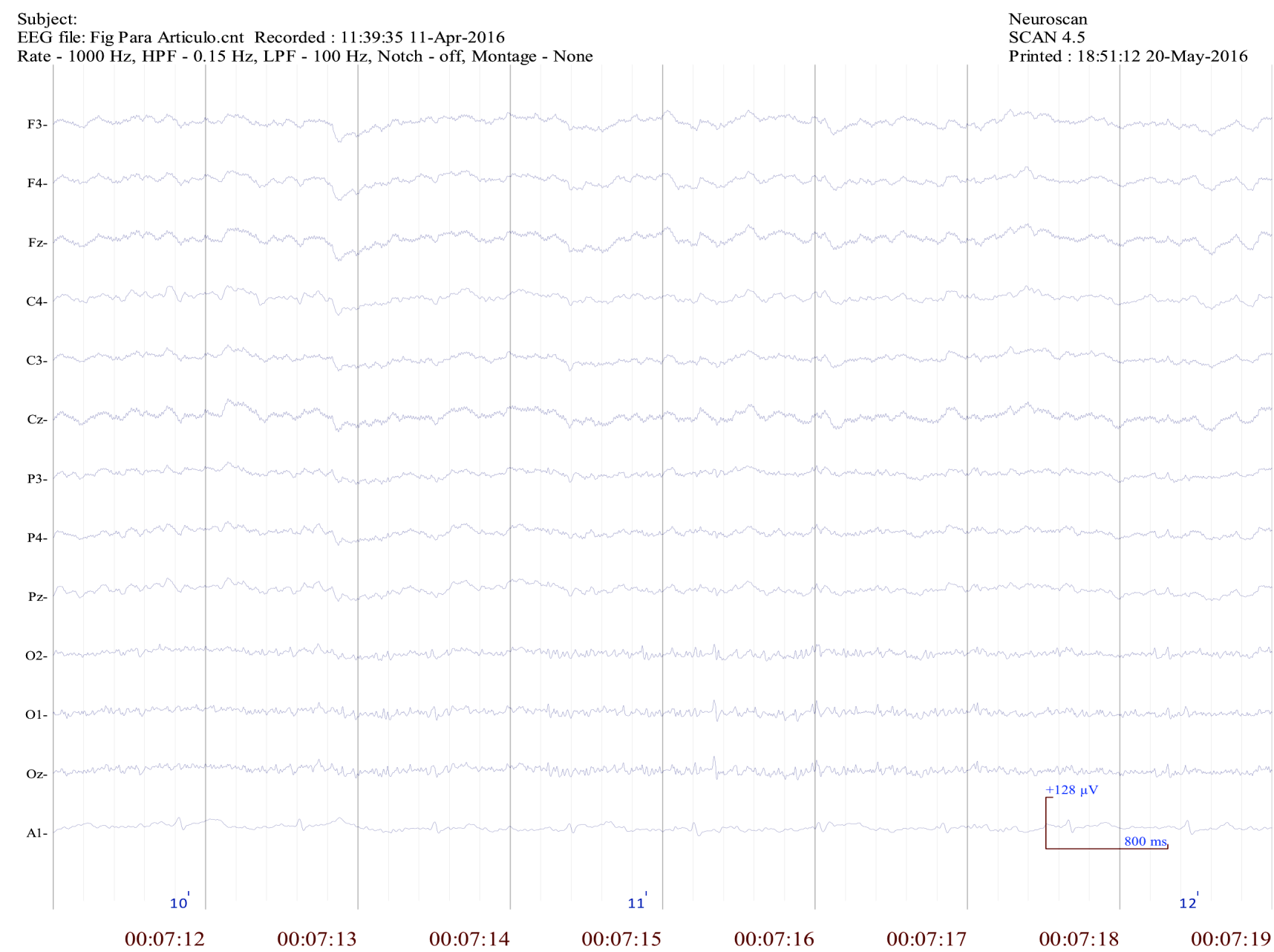

FIGURA 1. Fragmento del registro electrofisiológico de un participante. Se registran los pulsos de inicio de la presentación de la figura ambigua, representada por el número 10, y secuencialmente aparecen las respuestas que da el participante señaladas con los números 11 y 12, para indicar un cambio en la percepción de la cara principal del cubo hacia alguno de los lados. Ver explicación en el apartado "Archivo conductual".

\section{METODOLOGÍA}

PercepFiguras se realizó en VISUAL C++ que es un lenguaje de programación de tercera generación. Su ventaja es su flexibilidad para programar de acuerdo a los requerimientosdeestainvestigación. PercepFiguras, permite sincronizar la presentación de los estímulos y respuestas de los participantes con los sistemas de registro electrofisiológico. En este programa es importante tener una adecuada precisión en el registro de la respuesta conductual y la actividad cerebral asociada, lo cual no siempre ha sido posible con los programas disponibles para este fin ${ }^{[19]}$.
PercepFiguras se desarrolló de acuerdo a los requerimientos de los sistemas de adquisición de registros electrofisiológicos que se emplean en la actualidad en los laboratorios de investigación (e.g NeuroSCAN). Corre en el sistema operativo Windows (XP o superior), con requerimiento mínimo de procesador Pentium 3.

El programa puede ser descargado del siguiente enlace (donde también se encuentra el manual del usuario):

http://www.mediafire.com/file/9izlheh5w8z7yxz/ PercepFiguras.rar 


\section{Características de PercepFiguras}

El programa se diseñó para permitir al usuario elaborar una tarea de presentación continua de estímulos visuales, definir la secuencia de presentación, incluir cualquier tipo de figuras, recibir múltiples respuestas a una misma figura y manipular la duración y el intervalo de presentación entre éstas.

PercepFiguras puede acoplarse con cualquier sistema de registro electrofisiológico que reciba pulsos TTL, enviando un pulso con estas características desde la computadora de estimulación al sistema de registro, en el preciso momento en que se presenta el estímulo. También se envía un pulso al momento en el que el participante emite cada respuesta (Figura 1). Al final de cada sesión experimental, PercepFiguras crea un archivo con las respuestas conductuales (Archivo conductual) que registra el tipo de respuestas y el tiempo entre ellas, para que sea posible acoplarlo, fuera de línea, con el registro electroencefalográfico obtenido.

Adicionalmente, para cada ejecución, el programa genera automáticamente un archivo que contiene el número total de cada tipo de respuestas para cada tipo de figura, con su respectivo promedio de latencias (archivo de totales y promedios).

\section{Funcionamiento del programa}

El programa requiere tener en el mismo directorio: el archivo ejecutable, el archivo "imagenes.txt", el archivo "secuencia.txt" y los subdirectorios de "sonidos" e "imagenes"1.

El subdirectorio "imagenes” aloja el archivo "figuras ambiguas.bmp“, que contiene nueve imágenes en una matriz de $4 \mathrm{X} 3$, con espacio para añadir tres más (Figura 2). Si se requiere incorporar más de tres figuras nuevas, pueden remplazarse las figuras ya incluidas.

'Se recomienda que los nombres de archivos y subdirectorios no incluyan acentos, para asegurar compatibilidad.
Cada renglón del archivo "imagenes.txt" contiene la etiqueta numérica y el nombre del archivo de imágenes, donde se encuentra la figura que habrá de presentarse, así como sus coordenadas de localización dentro de ese archivo. Los detalles y procedimientos para definir estos datos se pueden consultar en el manual del usuario.

En la Figura 3a se muestra un ejemplo del contenido del archivo "imagenes.txt", en el que se utilizan tres figuras: el cubo ambiguo al que se le dio la etiqueta ("10"), el cubo con la cara principal a la izquierda (“20") y el cubo con la cara principal a la derecha (“30”), indicándose la ruta de ubicación y las coordenadas (últimas dos columnas) según el arreglo de imágenes de la Figura 2.

El archivo "secuencia.txt" contiene la secuencia de todas las figuras que se presentan en el experimento, separadas en bloques de 12, por una pantalla de descanso. Contiene además, el tipo de figura y su duración en milisegundos.

En la Figura 3b se presenta un fragmento de este archivo. El primer renglón indica el número de figuras a presentarse antes de una pantalla de descanso.

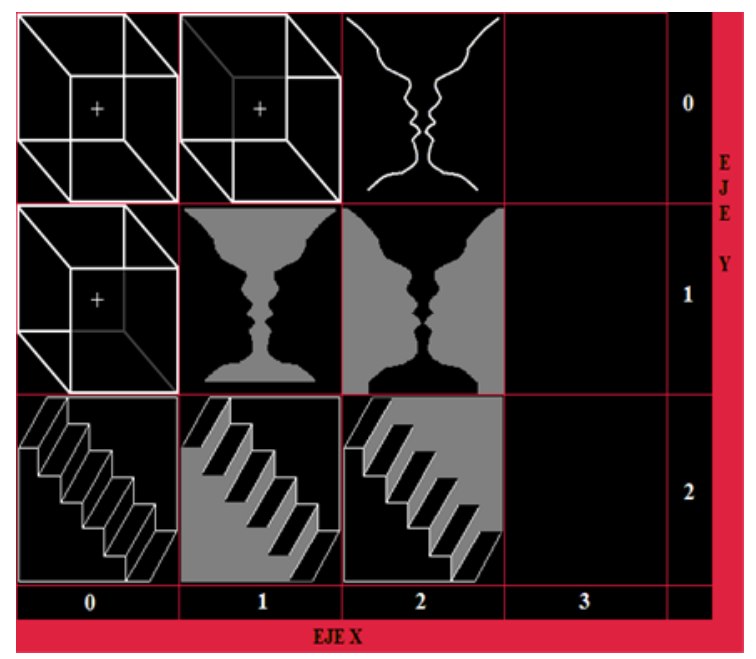

FIGURA 2. "imagenes". Muestra nueve figuras ambiguas con sus coordenadas $(x, y)$ y espacios vacíos para añadir tres figuras adicionales. 
Este número puede cambiarlo el usuario. Después, cada renglón contiene un número secuencial por cada ensayo comenzando con el 1, el tipo de figura y su duración en milisegundos.
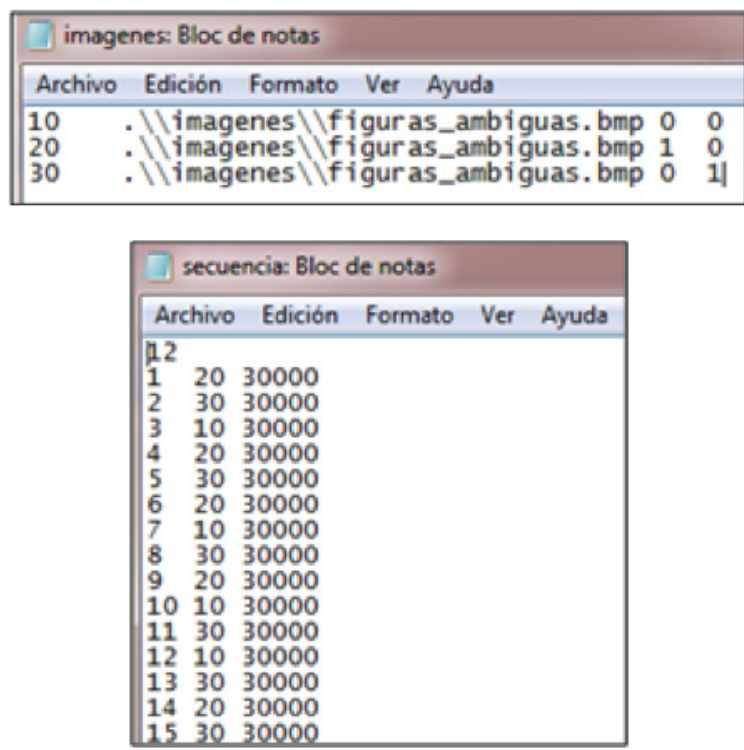

b)

FIGURA 3. Ejemplo de creación de archivos.

a) es el archivo de figuras, donde se especifica la etiqueta de las figuras, su ruta de acceso y las coordenadas $(x, y)$ en el archivo de imágenes.

b) es el archivo de secuencia, donde se definen las características de presentación del estímulo.

Una vez definidos los archivos de imágenes y de secuencia, se puede correr el programa PercepFiguras. Al inicio se muestra una pantalla de menú con tres opciones: PRÁCTICA, permite correr la tarea sin guardar nada en memoria, para que el usuario pueda familiarizarse con la tarea que habrá de ejecutar. En la opción PRUEBA se realiza el experimento; en ésta el programa guarda en el disco duro los resultados conductuales en un archivo llamado "Archivo Conductual". Finalmente, la opción SALIR termina el programa.

El experimento consiste en presentar una secuencia de imágenes en donde el participante deberá responder cada vez que cambie su percepción, con las teclas de navegación (flechas izquierda y derecha) represen- tadas en el archivo conductual como 1, si en respuesta a la presentación del estímulo se presiona la tecla flecha izquierda y 2 si se presiona la tecla flecha derecha.

\section{Archivo Conductual}

Como ya se dijo, al finalizar la tarea PercepFiguras crea un archivo conductual con el nombre "usuario.txt" (Figura 4). Cada vez que éste se ejecute, debe guardarse con otro nombre para evitar que se sobrescriba.

\begin{tabular}{|c|c|c|c|c|c|}
\hline \multicolumn{6}{|c|}{ Archivo Edición Formato Ver Ayuda } \\
\hline Trial & Resp & Type & correct & Latency & stim/Resp \\
\hline 1 & 0 & 20 & 1 & 1065 & stim \\
\hline$\overline{2}$ & 2 & 22 & 1 & 3005 & Stim \\
\hline 3 & 1 & 21 & 1 & 10137 & stim \\
\hline 4 & $\overline{2}$ & 22 & 1 & 3286 & stim \\
\hline 5 & 1 & 21 & 1 & 5633 & stim \\
\hline 6 & $\overline{2}$ & $2 \overline{2}$ & 1 & 7595 & stim \\
\hline 7 & 0 & 30 & 1 & 721 & stim \\
\hline 8 & 1 & 31 & 1 & 815 & stim \\
\hline 9 & $\overline{2}$ & 32 & 1 & 10169 & stim \\
\hline 10 & 1 & 31 & 1 & 2567 & stim \\
\hline 11 & $\overline{2}$ & $3 \overline{2}$ & 1 & 7510 & stim \\
\hline 12 & 1 & 31 & 1 & 4256 & stim \\
\hline 13 & 2 & 32 & 1 & 5716 & stim \\
\hline 14 & 0 & 10 & 1 & 1754 & stim \\
\hline 15 & 2 & 12 & 0 & 1628 & stim \\
\hline 16 & 1 & 11 & 1 & 14486 & stim \\
\hline 17 & 1 & 11 & 0 & 7791 & stim \\
\hline 18 & $\overline{2}$ & 12 & 1 & 4781 & stim \\
\hline 19 & 0 & 20 & 1 & 440 & stim \\
\hline 20 & 1 & 21 & 1 & 7603 & stim \\
\hline 21 & 2 & 22 & 1 & 5883 & stim \\
\hline 22 & 1 & 21 & 1 & 5163 & stim \\
\hline 23 & $\overline{1}$ & 21 & 0 & 9261 & stim \\
\hline 24 & $\overline{2}$ & 2 & 1 & 4217 & stim \\
\hline 25 & 0 & 3 & 1 & 2567 & st im \\
\hline
\end{tabular}

FIGURA 4. Archivo conductual de PercepFiguras.

En este archivo aparecen los datos sobre la ejecución de cada participante. La columna "Trial" se refiere al número de ensayo, "Resp" indica el tipo de respuesta que se dio, mientras que "Type" dice el tipo de figura al que perteneció esa respuesta. Por su parte la columna "Correct" indica si ésta fue correcta o no; en "Latency" aparece el tiempo que hubo entre una respuesta y otra.

El significado para cada columna es el siguiente:

La primera columna, “Trial” es una serie de números consecutivos a partir de 1 que indica la aparición de una figura y/o una respuesta.

La columna "Resp" será o cuando ocurra la aparición de una figura, 1 si la respuesta se hizo con la tecla flecha izquierda, y 2 si se presionó la tecla flecha derecha. 
En la columna "Type" se muestra la etiqueta que se asignó a la figura presentada (ejemplo: 10, 20, o 30). Si hubo respuestas a esa figura en "Resp", el programa pondrá en "Type" el número de la etiqueta correspondiente a la figura, más 1 si la respuesta fue izquierda o más 2 si fue derecha (ejemplo: figura 10 con respuesta 1 será 11 , figura 30 con respuesta 2 será 32, etc.). Si no hay ninguna respuesta, no se genera ningún renglón y aparece el código de la siguiente figura.

La columna "Correct" evalúa la respuesta dada en "Resp", y será correcta ("1") siempre que sea opuesta a la inmediata anterior, es decir si la respuesta en un ensayo fue hecha con la tecla izquierda (código 1), en el siguiente deberá ser hecha con la tecla derecha (código 2), porque el sujeto solo debe responder si percibe un cambio de orientación en la cara principal de la figura. En el inicio de cada bloque, la primera respuesta siempre será correcta.

En la columna "Latency", se registra el tiempo transcurrido en milisegundos desde la aparición de una figura hasta la respuesta que se le da, o desde una respuesta a la siguiente, no importa que haya otra figura entre las dos respuestas (ver Figura 5, intervalo a). Para la última respuesta de un bloque, la latencia será el tiempo desde esta respuesta hasta terminar la presentación del bloque (ver Figura 5, intervalo b).
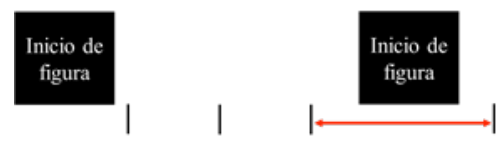

a)

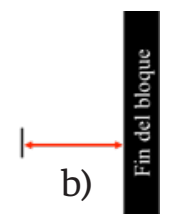

FIGURA 5. Muestra de los casos del último intervalo de respuesta a una figura. Las marcas verticales corresponden a las respuestas del participante.

La columna "Stim/Resp" es una columna adicional, sin significado para el programa, pero que se agregó para que el archivo conductual quedara tal como lo crea el programa STIM.

\section{Archivo de totales y promedios}

Este archivo se construyó para ofrecer un resumen de la ejecución conductual de los sujetos. Los datos que contiene son: el número total de respuestas izquierdas y derechas para cada tipo de figura (sección "frecuencias", figura 6) en todo el experimento, así como el promedio de las latencias de las mismas. La Figura 6 presenta un ejemplo de este archivo.

Las “frecuencias” son el número de respuestas correctas específicas (percepción izquierda o derecha) a cada una de las figuras etiquetadas como 10, 20, o 30. La respuesta a la primera figura de cada bloque no cuenta, ya que no es un cambio espontáneo en la percepción.

En la sección "promedios” se presenta el promedio en milisegundos de las latencias de las respuestas correctas dadas a cada tipo de figura por separado. A diferencia de la "frecuencia", en el cálculo del promedio de las latencias, sí se toma en cuenta el tiempo de la primera respuesta, porque el promedio refleja la duración total de la percepción y no del cambio de la percepción. Este archivo puede exportarse a un paquete de análisis estadístico para un análisis más completo.

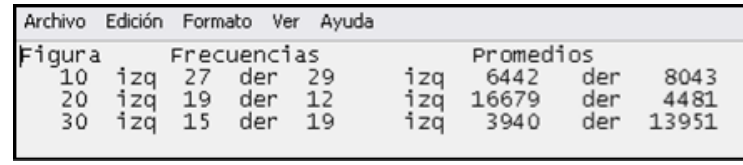

FIGURA 6. Archivo de totales y promedios.

\section{Solución al problema de la precisión del tiempo}

Uno de los principales retos fue lograr precisión en la medición del tiempo de duración de los estímulos y de los tiempos de respuesta. Esto se resolvió calibrando al programa en la computadora de estimulación, con ayuda de un dispositivo externo, en este caso el equipo de registro electrofisiológico NeuroSCAN.

El programa utiliza de manera interna ciclos con una duración fija, que se logra colocando al principio del 
ciclo una instrucción que accede al valor interno del timer (ver la instrucción de la Figura 7 "lee tiempo inicial del ciclo en el timer"); y al final de cada ciclo, otra instrucción que compara su valor actual con el primero, para asegurar que el tiempo transcurrido del ciclo sea siempre el mismo (ver la condición de la Figura 7 “¿ya pasaron 33 milisegundos?”). Para lograr que un estímulo tenga una duración " $\mathrm{x}$ ” en segundos, se divide " $x$ " entre la duración de un ciclo, dando como resultado el número total de ciclos que debe durar el estímulo.

Usando el procedimiento anterior el estímulo debería tener la duración esperada, pero en la práctica esto se comprueba corroborando con un instrumento externo. En el presente caso, la precisión del tiempo de duración de los estímulos se corroboró mandando una marca a los registros de Neuroscan tanto al momento de inicio como de finalización de la presentación del estímulo visual, para posteriormente medir el tiempo transcurrido entre dichas marcas. Un ejemplo de este procedimiento se puede observar en la figura 1: para un estímulo al que se dio una duración de 30 segundos, aparece sobre el registro electrofisiológico la marca " $10 "$ que indica el inicio del estímulo en el minuto 00:07:12 (7 minutos con 12 segundos); el segundo pulso, que indica su finalización (no observable en la Figura 1) apareció en el minuto 00:07:42, lo que permite corroborar que el tiempo de duración del estímulo fue el deseado (30 segundos: 00:07:1200:07:42). De la misma forma, para evaluar la precisión en la medición de la latencia de las respuestas de los participantes, se procedió a medir en el registro electrofisiológico la diferencia entre las marcas de la respuesta ante un cambio perceptual y el siguiente cambio. Es decir, como se puede observar en la figura 1, la primera respuesta que dio el participante al estímulo "10" apareció al minuto 00:07:15, representada con la marca "11" y una segunda respuesta (marca "12") fue dada en el minuto 00:07:18:5, lo que nos indica que el tiempo transcurrido entre las dos respuestas fue de 3 segundos con 500 milisegundos. Con el procedimiento anterior, se obtuvieron valores similares entre el cálculo hecho con PercepFiguras en el archivo conductual y la medición hecha con el aparato de registro externo. Aunque hubo pequeñas diferencias en algunas mediciones en su mayoría no superaron los 10 milisegundos.

\section{Diagrama de flujo del programa PercepFiguras}

La Figura 7 muestra el diagrama de flujo del programa PercepFiguras para el diseño de un bloque de 12 figuras de cubos, con duración de 30 segundos cada uno. De acuerdo al diseño de la tarea, el arreglo "iCUBOS" consta de 12 elementos que pueden ser una de tres figuras posibles: cubo ambiguo, cubo inductor izquierdo o cubo inductor derecho.

"Nciclos" es un índice que cuenta el número de ciclos, cada uno con una duración fija de $33 \mathrm{~ms}$, por lo que se necesitan 900 ciclos para dar a cada cubo la duración aproximada de 30 segundos (29.9 s). En la instrucción "lee tecla" se puede tener tecla derecha y tecla izquierda. Más adelante cuando el sujeto responde, se manda el pulso de tecla, que envía al equipo de registro el código de respuesta, que corresponde a la columna "Type" del archivo conductual.

\section{Estudio piloto}

Con el objetivo de probar el programa PercepFiguras, se realizó un estudio piloto.

\section{Tarea}

Se diseñó una tarea en la que se presentaron 4 bloques con 12 cubos cada uno. La duración de cada cubo fue de 30 segundos, así cada bloque de presentación tuvo una duración de 6 minutos, que es el tiempo óptimo para evitar artefactos durante el registro electrofisiológico ${ }^{[13]}$.

Las características de la tarea fueron las siguientes: 


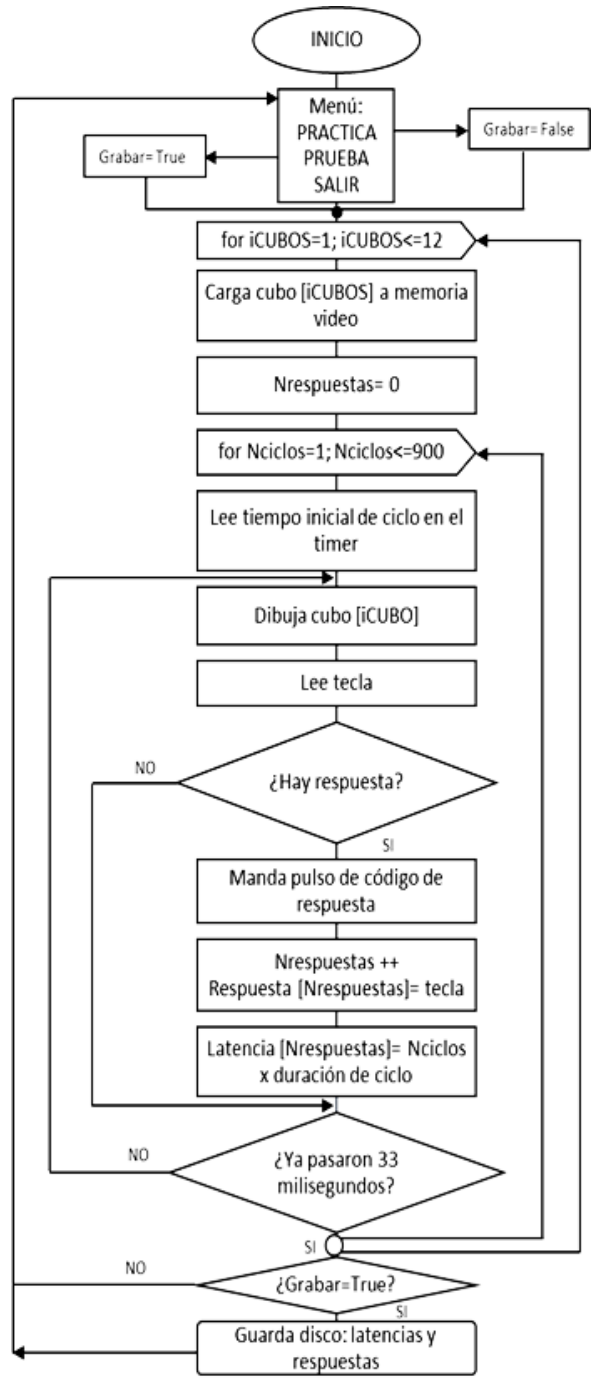

FIGURA 7. Diagrama de flujo del programa PercepFiguras.

1) Se seleccionaron tres tipos de cubos: un cubo ambiguo y dos cubos controles inducidos hacia el lado derecho o lado izquierdo: cubo izquierdo y cubo derecho (los tres cubos de la Figura 2).

2) En cada bloque, ninguna de las figuras se presentó dos o más veces seguidas.

3) La secuencia de figuras se presentó sin ningún evento intermedio entre ellas.

4) En cada bloque, cada uno de los cubos se presentó 4 veces.
5) Cada bloque tenía diferente secuencia de presentación. Al terminar cada bloque aparecía una pantalla de descanso, excepto en el último, en el que aparecía una pantalla de fin del experimento.

Se instruyó a los participantes para que respondieran cada vez que cambiara su percepción de la figura observada, presionado una de dos teclas posibles: la flecha izquierda cuando percibían la orientación de la cara principal del cubo hacia la izquierda y la flecha derecha cuando la percibían hacia la derecha.

\section{Hipótesis}

De acuerdo con los resultados de estudios previos ${ }^{[22]}$ se plantearon 3 hipótesis, que de ser apoyadas en el presente estudio, mostrarían la utilidad del programa para el estudio de la percepción multiestable.

a) El cubo ambiguo tendrá mayor número de cambios perceptuales con relación a los cubos control. En consecuencia, el tiempo de percepción estable (estabilidad) del cubo ambiguo será menor.

b) Asimismo, se espera que la mayoría de los cambios perceptuales en las figuras control inductoras sea en la dirección del cambio perceptual inducido, en comparación con la dirección opuesta. Para el cubo ambiguo, no se esperan diferencias en los cambios perceptuales entre la orientación izquierda y derecha.

c) En cuanto a la duración de la percepción, se espera que los cubos control inductores muestren mayor estabilidad en la dirección del cambio perceptual inducido (izquierdo o derecho). Es decir, se espera que una vez que se dé el cambio perceptual hacia el lado inducido, los individuos mantengan por más tiempo esta percepción, antes de que se produzca un nuevo cambio. En el caso del cubo ambiguo no se esperan diferencias en la estabilidad de la percepción hacia izquierda o derecha. 


\section{Material y método}

Participaron 15 estudiantes universitarios (5 hombres), con una edad promedio de 20.6 años y desviación estándar de 3.14 años. La muestra fue seleccionada de manera no probabilística, la participación de todos fue voluntaria y fueron informados sobre los objetivos del estudio.

La prueba se realizó en un cuarto libre de distractores, la presentación del programa se hizo en una computadora con sistema operativo XP con un monitor de 19". El tamaño de los estímulos fue de $6.8 \mathrm{~cm}$ con un ángulo visual de $6^{\circ}$ a una distancia de $62 \mathrm{~cm}$ entre el participante y el monitor. Al inicio de la prueba se pidió a los participantes que durante cada bloque, trataran de mantener la mirada en el punto de fijación de la figura.

\section{Análisis estadístico}

Se realizó un análisis de la varianza (ANOVA) de medidas repetidas de 2 factores para comparar la frecuencia de cambios y la estabilidad de la percepción por separado: factor Condición con 3 niveles (figuras ambigua, izquierda y derecha) y factor Dirección del cambio perceptual (Dcp) con 2 niveles (cambio izquierdo y cambio derecho). Se aplicó la prueba de esfericidad W de Mauchly; en caso de que no pasaran la prueba, se reportaron los valores de la corrección de Huynh-Feldt ( $\varepsilon$ Huynh-Feldt). Adicionalmente se obtuvieron los indicadores del tamaño del efecto (n2p). En los análisis posthoc se utilizó la prueba de Bonferroni. Para comprobar la distribución normal de los datos, se realizó la prueba de Kolmogorov-Smirnov.

\section{RESULTADOS Y DISCUSIÓN}

\section{Resultados conductuales}

Para el análisis de los datos solo se tomaron en consideración las respuestas correctas, es decir, aquellas que indicaban que el participante había experimentado un cambio en la percepción. Se analizó la frecuencia de cambios perceptuales espontáneos por condi- ción (cubo ambiguo, cubo derecho y cubo izquierdo), además se obtuvo el promedio de latencias, para medir el tiempo en que permaneció estable la percepción en una condición determinada.

El 85\% de las 12 variables pasaron la prueba de normalidad aplicada, mostrando una probabilidad mayor a 0.05 .

Con relación a la frecuencia de cambios perceptuales, se encontraron efectos principales entre las condiciones $[\mathrm{F}(2,28)=16.52, \mathrm{p}<0.001, \mathrm{\eta} 2 \mathrm{p}=0.541]$. El promedio de la frecuencia de cambios en cada presentación del cubo ambiguo fue de 5 cambios en 30 segundos. De acuerdo a los análisis Posthoc, el cubo ambiguo tuvo en total un mayor número de cambios (Media=77, $\mathrm{SD}=42)$ respecto al cubo izquierdo (Media=53, $\mathrm{SD}=37$ ) (DMTukey=24.26, $\mathrm{p}<0.05)$ y respecto al cubo derecho (Media=40, SD=32) (DMTukey= 36.66, $\mathrm{p}<0.001)$. Asimismo, el cubo izquierdo tuvo significativamente mayor número de cambios con relación al cubo derecho (DMTukey=12.4, $\mathrm{p}<0.05)$.

Además, se encontró una interacción significativa Condición x Dcp, $[\mathrm{F}(1.2,18.9)=62.7, \mathrm{p}<0.0001$, $\varepsilon$ Huynh-Feldt=0.677, n2p=0.818]. De acuerdo a los análisis Posthoc (Figura 8), la figura ambigua presentó más cambios perceptuales derechos que izquierdos (Media=40, DS=20 vs Media=38, DS=21) (DMTukey=2.2, $\mathrm{p}<0.001)$. En la figura izquierda los cambios perceptuales izquierdos fueron más que los cambios derechos (Media=30, DS=17 vs Media=23, DS=19) (DMTukey=7.8, $\mathrm{p}<0.001$ ) y en la figura derecha se observaron más cambios perceptuales derechos que izquierdos (Media = 23, DS=15 vs Media=18, DS=17) (DMTukey=5.3, $\mathrm{p}<0.001)$.

En cuanto al análisis de la estabilidad de la percepción, se observaron efectos principales entre las condiciones $[\mathrm{F}(2,28)=14.19, \mathrm{p}<0.001, \eta 2 \mathrm{p}=0.503]$; el cubo ambiguo mostró menor estabilidad de la percepción (Media $=6787 \mathrm{~ms}, \mathrm{SD}=3052 \mathrm{~ms}$ ) comparado con el cubo 


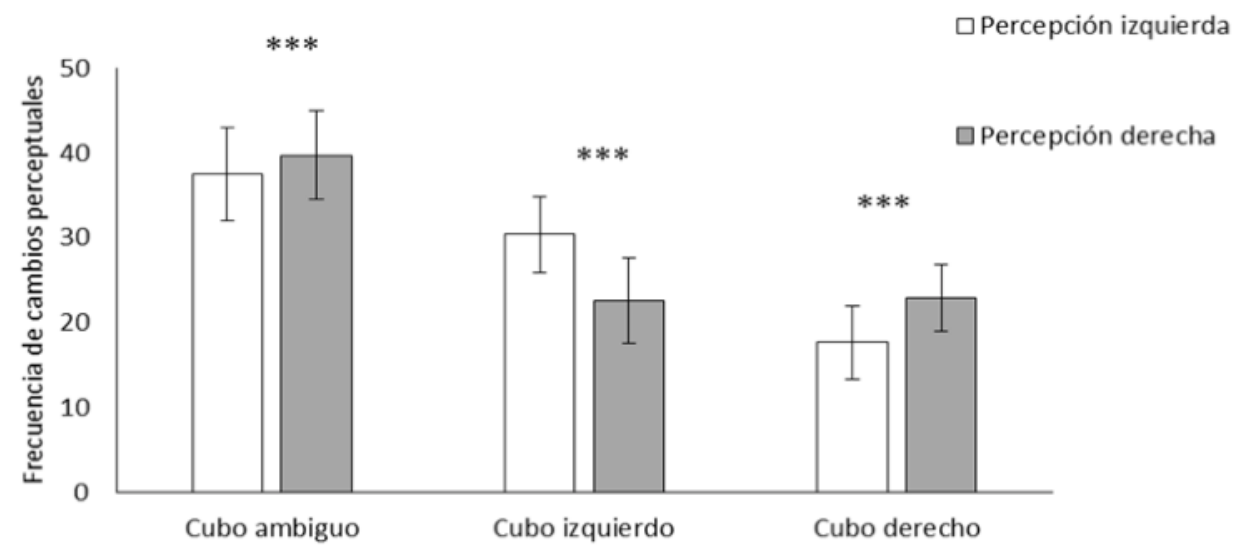

FIGURA 8. Comparación del número de cambios perceptuales entre condición para cada tipo de dirección del cambio perceptual. ${ }^{* * *}=\mathrm{p} \leq 0.001$. Las líneas verticales en las barras señalan el error estándar.

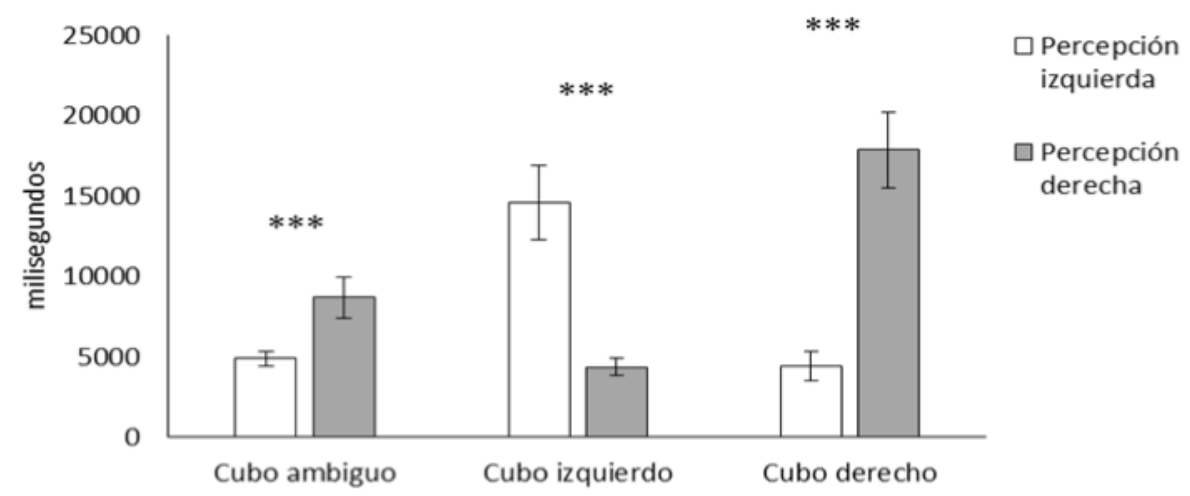

FIGURA 9. Comparación del tiempo (milisegundos) de estabilidad perceptual entre condición para cada tipo de dirección del cambio perceptual. ${ }^{* * *} \mathrm{p} \leq 0.001$. Las líneas verticales en las barras señalan el error estándar.

izquierdo (Media=9484ms, SD=4,744ms) (DMTukey= 2697, $\mathrm{p}<0.01$ ) y con el cubo derecho (Media=11158ms, $\mathrm{DS}=5032$ ) (DMTukey=4371, $\mathrm{p}<0.001$ ); sin embargo, las diferencias entre los cubos inductores izquierdo y derecho no fueron significativas (DMTukey=1674, $\mathrm{p}=0.14$ ).

Por otra parte, se observó una interacción significativa Condición x Dcp $[F(1,16)=23.3, p<0.0001$, عHuynhFeldt $=0.574, \eta 2 p=0.625]$. Como se muestra en la Figura 9 y de acuerdo a los análisis Posthoc, en el cubo ambiguo la percepción derecha duró más tiempo que la percepción izquierda (Media $=8700 \mathrm{~ms}, \mathrm{DS}=4897 \mathrm{~ms}$ vs Media=4872, DS=1834）（DMTukey=1078.3, p<0.0001).
En el cubo izquierdo la estabilidad de la percepción izquierda fue mayor que la percepción derecha (Media= $14596 \mathrm{~ms}$, DS=9047ms vs Media=4371ms, DS= 1973ms) (DMTukey=2330.5, $\mathrm{p}=0.003$ ); mientras que en el cubo derecho se mantuvo más tiempo la percepción derecha en comparación con la izquierda (Media $=17891 \mathrm{~ms}, \mathrm{DS}=$ 9183ms vs Media=4424ms, DS=3566ms) (DMTukey= 2487.3, $\mathrm{p}<0.0001)$.

\section{Discusión}

En la mayoría de los estudios de percepción son poco comunes los estudios que evalúen las respuestas del individuo en ausencia de variaciones en el ambiente. 
Sin embargo, estos métodos son empleados cuando se estudian fenómenos específicos de ilusiones perceptuales en sus diferentes modalidades sensoriales.

El objetivo del presente trabajo fue desarrollar el programa PercepFiguras como una herramienta en el estudio conductual y electrofisiológico de la percepción visual multiestable. El programa admite múltiples respuestas ante la presentación de un mismo estímulo, así como la medición precisa de los tiempos de presentación de los estímulos y delos tiempos de respuesta de los participantes; además de la sincronización con programas de registro electrofisiológico.

Los tiempos en los que ocurrieron los estímulos y respuestas, proporcionados por PercepFiguras, se compararon con técnicas de registro externo, lo que hizo posible ajustar la duración de los ciclos para obtener una mayor precisión. En comparación con los tiempos registrados con el aparato de registro externo se observaron algunas diferencias con los registrados con PercepFiguras; sin embargo, éstas fueron tan pequeñas que puede considerarse que PercepFiguras presenta una precisión bastante aceptable para tales estudios.

Para validar la utilidad de PercepFiguras, se consideró comparar los resultados obtenidos en el estudio piloto con lo reportado en trabajos previos ${ }^{[21]}$. Los resultados del presente estudio mostraron el patrón de respuestas que se planteó en la primera hipótesis: mayores cambios perceptuales en la figura ambigua respecto a las figuras control inductoras. Aunque el número reducido de estudios que utilizan figuras control en un diseño de presentación continua hace difícil comparar nuestros resultados con los de la literatura, los resultados obtenidos con PercepFiguras se comportaron de acuerdo a lo que se puede esperar en este tipo de estudios.

El promedio de la frecuencia de cambios en el cubo ambiguo en este estudio, fue menor al que se reporta en otras investigaciones (9 vs 15 cambios por minuto) ${ }^{[4]}$.
Esta diferencia podría estar asociada con la mayor duración con la que se presentan los estímulos en la mayoría de los trabajos consultados, ya que a mayor tiempo de observación de la figura, se presentan mayor número de eventos oculares, que pueden inducir más cambios perceptuales, lo cual de ninguna manera es deseable cuando se realizan registros electrofisiológicos.

Asimismo, como se planteó en la segunda hipótesis, las figuras control tuvieron una mayor frecuencia hacia el lado inducido. Resultados similares se han obtenido en otras investigaciones donde los participantes detectan correctamente el 95\% de los cambios inducidos ${ }^{[13]}[22]$. No obstante, el cubo ambiguo también tuvo mayores cambios significativos hacia la dirección derecha de la percepción, aunque en menor proporción que en las figuras control.

Con relación a la tercera hipótesis, los resultados obtenidos la apoyaron parcialmente, ya que si bien es cierto que ambas figuras control mostraron mayor duración en la percepción hacia el lado inducido, también hubo cambios significativos en la duración de la percepción hacia uno de los lados en la figura ambigua, no obstante, estas diferencias fueron menores que las ocurridas en las figuras control.

Tomando en cuenta los resultados anteriores, puede decirse que en general los resultados concuerdan con lo observado en estudios similares al presente ${ }^{[4]}$, por lo que una vez más es posible afirmar que PercepFiguras puede ser útil para el estudio de los fenómenos conductuales y electrofisiológicos de la percepción multiestable.

Desde el punto de vista psicológico, el hecho de encontrar algunas diferencias entre los resultados obtenidos y lo esperado en la segunda y tercera hipótesis, evidencia una preferencia en mantener la percepción hacia la derecha en el cubo ambiguo y los cubos 
control. Este resultado podría relacionarse con la manera en que aprendemos a interpretar los objetos que aparecen con mayor frecuencia en el ambiente ${ }^{[23]}$ [24]. La configuración espacial de la cara principal derecha del cubo se encuentra orientada hacia abajo, por lo que puede interpretarse como un objeto que está descansando sobre una superficie. En comparación las figuras cuya configuración es similar a la de la cara izquierda del cubo, que se encuentra orientada hacia arriba, dan la apariencia de un cubo que flota, lo que es menos común observar en un ambiente natural.

\section{CONCLUSIONES}

Tomando en consideración los resultados en conjunto, se podría decir que las diferencias encontradas entre la frecuencia de cambios y el tiempo de estabilidad perceptual entre figuras ambiguas y control, dan cuenta de que los cambios perceptuales pueden ser claramente influenciados por la modificación de las características físicas de los estímulos, apoyando así el modelo de aproximación "bottom-up" que atribuye un papel importante a la influencia de las rasgos sensoriales de los objetos sobre la percepción multiestable ${ }^{[25]}$.
No obstante, a pesar de que los estímulos control inducen la percepción hacia un tipo de orientación, el hecho de seguir observando cambios perceptuales hacia el lado contrario al inducido, da cuenta de que la percepción multiestable se encuentra influenciada también por procesos intrínsecos del sistema perceptual (conocidos como "top-down") ${ }^{[6]}$.

A pesar de que existe bastante información acerca del fenómeno de percepción multiestable en adultos jóvenes sanos, su estudio se ha abordado poco en diferentes poblaciones, por lo que se propone estudiarlo en diferentes grupos de edad, en poblaciones clínicas con trastornos perceptuales (esquizofrenia, demencia, autismo, etc.) o simplemente con alguna característica psicológica (coeficiente intelectual, personalidad, creatividad, etc.).

\section{AGRADECIMIENTOS}

Este trabajo es parte de un proyecto de doctorado apoyado por una beca del Consejo Nacional de Ciencia y Tecnología (480412/335718). Los autores declaran no tener ningún conflicto de intereses. 


\section{REFERENCIAS}

[1] Orlandi N. Visual switching: the illusion of instantaneity and visual search. Rev Philos Psychol. Springer; 2012;3(4):469-80. DOI 10.1007/s13164-012-0098-z

[2] Necker LA. LXI. Observations on some remarkable optical phænomena seen in Switzerland; and on an optical phænomenon which occurs on viewing a figure of a crystal or geometrical solid. London Edinburgh Philos Mag J Sci. Taylor \& Francis; 1832;1(5):329-37.

[3] Kogo N, Hermans L, Stuer D, van Ee R, Wagemans J. Temporal dynamics of different cases of bi-stable figure-ground perception. Vision Res. Elsevier; 2015;106:7-19. http://dx.doi.org/10.1016/j.visres.2014.10.029

[4] Leopold DA, Wilke M, Maier A, Logothetis NK. Stable perception of visually ambiguous patterns. Nat Neurosci. Nature Publishing Group; 2002;5(6):605-9. DOI: 10.1038/nn851

[5] Kornmeier J, Hein CM, Bach M. Multistable perception: when bottom-up and top-down coincide. Brain Cogn. Elsevier; 2009;69(1):138-47. doi:10.1016/j.bandc.2008.06.005

[6] Intaitè M, Noreika V, Šoliūnas A, Falter CM. Interaction of bottom-up and top-down processes in the perception of ambiguous figures. Vision Res. Elsevier; 2013;89:24-31. http://dx.doi.org/10.1016/j.visres.2013.06.011

[7] Basar-Eroglu C, Mathes B, Khalaidovski K, Brand A, Schmiedt-Fehr C. Altered alpha brain oscillations during multistable perception in schizophrenia. Int J Psychophysiol. Elsevier; 2016;103:118-28. http://dx.doi.org/10.1016/j.ijpsycho.2015.02.002

[8] Ehm W, Bach M, Kornmeier J. Ambiguous figures and binding: EEG frequency modulations during multistable perception.

Psychophysiology. Wiley Online Library; 2011;48(4):547-58. DOI: 10.1111/j.1469-8986.2010.01087.x

[9] Kornmeier J, Bach M. Object perception: when our brain is impressed but we do not notice it. J Vis. Association for Research in Vision and Ophthalmology; 2009;9(1):7. doi:10.1167/9.1.7.

[10] Kornmeier J, Ehm W, Bigalke H, Bach M. Discontinuous presentation of ambiguous figures: How interstimulus interval durations affect reversal dynamics and ERPs. Psychophysiology. Wiley Online Library; 2007;44(4):552-60. DOI: $10.1111 / \mathrm{j} .1469-8986.2007 .00525 . x$

[11] Pearson J, Brascamp J. Sensory memory for ambiguous vision. Trends Cogn Sci. Elsevier; 2008;12(9):334-41. doi:10.1016/i.tics.2008.05.006

[12] Nakatani H, van Leeuwen C. Transient synchrony of distant brain areas and perceptual switching in ambiguous figures. Biol Cybern. Springer; 2006;94(6):445-57. DOI 10.1007/s00422-006-0057-9

[13] Ozaki TJ, Sato N, Kitajo K, Someya Y, Anami K, Mizuhara H, et al. Traveling EEG slow oscillation along the dorsal attention network initiates spontaneous perceptual switching. Cogn Neurodyn. Springer; 2012;6(2):185-98. DOI 10.1007/s11571-012-9196-y
[14] Nakatani H, Orlandi N, van Leeuwen C. Precisely timed oculomotor and parietal EEG activity in perceptual switching. Cogn Neurodyn. Springer; 2011;5(4):399-409. DOI 10.1007/s11571-011-9168-7

[15] Nakatani H, van Leeuwen C. Individual differences in perceptual switching rates; the role of occipital alpha and frontal theta band activity. Biol Cybern. Springer; 2005;93(5):343-54. https://doi.org/10.1007/s00422-005-0011-2

[16] Nakatani H, van Leeuwen C. Antecedent occipital alpha band activity predicts the impact of oculomotor events in perceptual switching. Front Syst Neurosci. Frontiers Media SA; 2013;7. doi: 10.3389/fnsys.2013.00019

[17] Gaetz M, Weinberg H, Rzempoluck E, Jantzen KJ. Neural network classifications and correlation analysis of EEG and MEG activity accompanying spontaneous reversals of the Necker cube. Cogn brain Res. Elsevier; 1998;6(4):335-46. https://doi.org/10.1016/S0926-6410(97)00038-4

[18] D. H. Brainard, D. G. Pelli MK. Psychophysics Toolbox. [Online]. http://www.psychtoolbox.org/HomePage, March. 2012;

[19] Plong M, Shen K, Van Vliet M, Robben A, Van Hulle M, Geurts L. Accurate visual stimulus presentation software for EEG experiments. Proceedings of the First Asian Conference on Information Systems. 2012.

[20] Sanz-Martin, A., Gumá-Díaz, E., Guevara, M. A., \& HernándezGonzález, M. MemPavox: prueba computarizada para evaluar la memoria de trabajo visual-verbal. Revista mexicana de ingeniería biomédica, 2014, vol. 35, no 1, p. 81-92.

[21] Toppino, T. C., \& Long, G. M. Time for a change: What dominance durations reveal about adaptation effects in the perception of a bi-stable reversible figure. Attention, Perception, \& Psychophysics, 2015, vol. 77, no 3, p. 867-882.

[22] Kornmeier J, Bach M. Early neural activity in Necker cube reversal: Evidence for low level processing of a gestalt phenomenon. Psychophysiology. Wiley Online Library; 2004;41(1):1-8. DOI: 10.1046/j.1469-8986.2003.00126.x

[23] Palmer SE. Vision science: Photons to phenomenology. MIT press Cambridge, MA; 1999.

[24] Hoffman DD, Menezo M. Inteligencia Visual: Cómo creamos lo que vemos. Paidós Ibérica; 2000.

[25] Kornmeier J, Bach M. Ambiguous figures-what happens in the brain when perception changes but not the stimulus. Front Hum Neurosci. Frontiers Media SA; 2012;6. doi: 10.3389/fnhum.2012.00051 